\title{
Experimental Study of Gravitation Effects on Liquid Crystal Phase Transitions in Polydisperse Aqueous Suspensions of $\mathrm{Mg}_{2} \mathrm{Al}$ Layered Double Hydroxide
}

\author{
Yun Chen ${ }^{1} \cdot$ Li Zhang ${ }^{1} \cdot$ Dejun Sun $^{1} \cdot$ Zhiwei Sun $^{2}$. \\ Shenghua $\mathrm{Xu}^{2}$
}

Received: 22 May 2015 / Accepted: 19 November 2015 / Published online: 2 December 2015

(C) Springer Science+Business Media Dordrecht 2015

\begin{abstract}
Effects of gravity on liquid crystal phase transitions (LCPT) in polydisperse aqueous suspensions of $\mathrm{Mg}_{2} \mathrm{Al}$ layered double hydroxide (LDHs) were studied under normal gravity condition. Samples with different suspension concentration (SC) were tested for 15 days and the relevant changes in samples were observed through crossed polarizers. Our results showed: (a) the samples were still isotropic (I) when SC $<23 \mathrm{wt} \%$; (b) when $23 \mathrm{wt} \%<\mathrm{SC}<$ $30 \mathrm{wt} \%$, a shear-induced birefringence appeared after preparation, and finally coexistence of four phases was reached, including an opaque isotropic top phase, a birefringent middle phase, a faint birefringence phase and a sediment layer of larger platelets resulting from the high polydispersity; (c) when $\mathrm{SC}>30 \mathrm{wt} \%$, the suspensions were in a gel state, and the gel network slowed down the LCPT. The above different behavior of phase transitions is apparently due to the concentration gradient and fractionation caused by gravity. This study provides guidance on how to select samples of LDHs suspensions for LCPT used in the upcoming experiment in the space program SJ-10 satellite.
\end{abstract}

Dejun Sun

djsun@sdu.edu.cn

1 Key Laboratory of Colloid and Interface Chemistry, Shandong University, Ministry of Education, Jinan 250100,

People's Republic of China

2 Key Laboratory of Microgravity, Institute of Mechanics, Chinese Academy of Sciences, Beijing 100190,

People's Republic of China
Keywords Gravity · Liquid crystal phase transition . Polydisperse suspension · Concentration

\section{Introduction}

Anisometric colloidal particles with high aspect ratio may be able to self-assemble into orientationally ordered liquid crystalline phases, involving nematic $(\mathrm{N})$, smectic $(\mathrm{S})$ and columnar (C) phases. Already in 1925, Zocher (1925) observed liquid crystal phase transition (LCPT) in the suspension of vanadium pentoxide particles for the first time. After that, the LCPT of non-spherical particles, such as rods and plates, has been attracting much attention (Landman et al. 2014; Hansen et al. 2013; Kleshchanok et al. 2012a). As indicated by Onsager (1949), the I-N transition is based on the fact that the loss of orientational entropy is smaller than the simultaneous gain of excluded volume entropy at high enough concentration. In fact, numerous studies have pointed out that lyotropic liquid crystals are highly affected by the gravitational force (Leferink Op Reinink et al. 2012, 2013). However, most of these findings were based on sedimentation tests carried out on the ground (Wijnhoven et al. 2005; Kleshchanok et al. 2012b) or computer simulations (Viveros-Méndez et al. 2014), and few of them could give an explanation to the influence of gravity by direct comparison of samples with and without the earth gravitational field due to the limitations of experimental conditions.

LCPT driven by gravity has been observed in many studies (Verhoeff et al. 2009; Vis et al. 2015). Under gravity, all particles will settle down to the bottom of the container, and the effect of entropy is to make all particles evenly distributed, thus the actual situation is controlled 


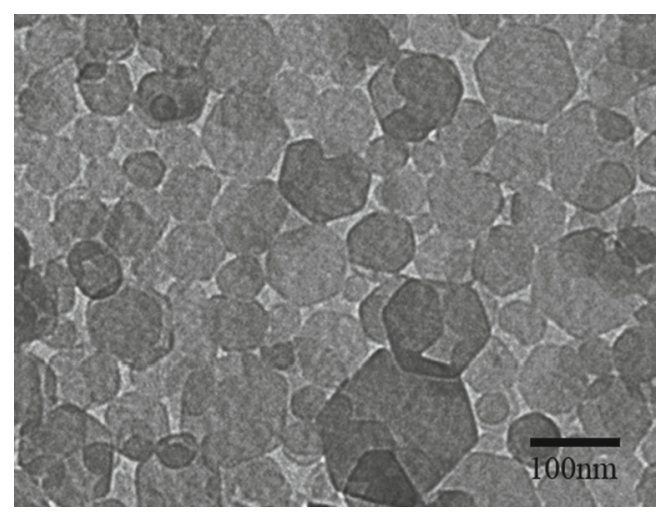

Fig. 1 Transmission electron micrograph of $\mathrm{Mg}_{2} \mathrm{Al}$ LDHs particles used in this study

by these two factors (Yang et al. 1977). After long time storage, the system reaches a sedimentation-diffusion equilibrium that arises from the balance between the gravity and the osmotic pressure. Using a simple model, Lekkerkerker (2004) qualitatively described this hydrostatic equilibrium that the osmotic pressure difference between two faces of each divided layer was balanced by the weight of the layer. Furthermore, the balance will cause a concentration gradient depending on the height in the cuvette (Kuijk et al. 2012), leading to multiple phases in one sediment. Veerman (1992) and Verhoeff (2012) found the suspension of hard platelets was able to show the N-C transition after the I-N transition in gravity at a high particle concentration.

Unlike highly monodisperse viruses (Lettinga et al. 2006), colloidal particles always exist a size distribution (Palberg 2014), which may suppress the formation of ordered structure (Vroege et al. 2006). For instance, if the polydispersity of colloidal rodlike particles is higher than $18 \%$, the smectic phase will not appear on the basis of computer simulation because the rods cannot fit into the smectic layers (Leferink Op Reinink et al. 2012). For suspensions of discotic particles, the computer simulation shows that the I$\mathrm{N}$ and $\mathrm{N}-\mathrm{C}$ transitions may appear for polydispersity below $17 \%$ (Veerman and Frenkel 1990; Bates and Frenkel 1998). However, researchers have recently indicated that for a polydisperse system, the fractionation induced by gravity could reduce the local polydispersity and further enrich the phase

Table 1 Characteristics of the colloidal platelets used in this study, as obtained by TEM (for the average diameter $<\mathrm{D}>$ ) and AFM (for the average thickness $<\mathrm{L}>$ )

\begin{tabular}{lllll}
\hline$<\mathrm{D}>/ \mathrm{nm}$ & $\sigma_{D} / \%$ & $<L>/ \mathrm{nm}$ & $\sigma_{L} / \%$ & $<D>/<L>^{a}$ \\
\hline 89 & 36 & 5.21 & 13 & 17 \\
\hline
\end{tabular}

${ }^{\mathrm{a}}<D>/<L>$ expresses the aspect ratio of the $\mathrm{Mg}_{2} \mathrm{~A} 1 \mathrm{LDHs}$ particles behavior of colloidal systems (Leferink Op Reinink et al. 2012; Byelov et al. 2010). Van den Pol (2008) showed that strong fractionation occurred in the highly polydisperse systems consisting of isotropic, nematic, smectic A and columnar phases. As noted by Fonseca (2009), such coexistence of multiple phases was due to the sedimentation-induced vertical distribution of particle fraction.

Layered double hydroxides (LDHs) are a typical class of inorganic compounds. The general formula for LDHs is:

$\left[\mathrm{M}_{1-\mathrm{X}^{2+}} \mathrm{M}_{\mathrm{X}}{ }^{3+}(\mathrm{OH})_{2}\right]^{\mathrm{X}+} \mathrm{A}^{\mathrm{n}-} \frac{\mathrm{x}}{\mathrm{n}}-\cdot \mathrm{mH}_{2} \mathrm{O}$

where $\mathrm{M}^{2+}$ and $\mathrm{M}^{3+}$ are the divalent and trivalent mental ion respectively, and $\mathrm{A}^{\mathrm{n}-}$ is the interlayer anion. The LDHs have a lamellar structure originating from the brucite-like structure of $\mathrm{M}^{2+}(\mathrm{OH})_{2}$, in which part of divalent metallic cations are replaced by trivalent ones. Nowadays, there are many studies about the application of LDHs, because they are easy to synthesize and be able to accommodate different ions (Zhang et al. 2013; Chowdhury, Bhattacharyya 2015). In our previous work, we have reported their ability of transition from a disordered isotropic phase to an orientationally ordered nematic (Liu et al. 2003) and lamellar (Wang et al. 2005) phase. In addition, the competition among sedimentation, nematic ordering and gelation was also studied based on ground test (Zhang et al. 2007).

SJ-10 program provides a mission of space microgravity experiments including both fields of space life science and microgravity science aboard the 24th recoverable satellite of China (Hu et al. 2014). This paper will report on a specific pilot study of LCPT for the upcoming experiment in the space program SJ-10 satellite, especially providing guidance on selecting samples of LDHs suspensions used for the program. Our experimental device on board will stay for only 15 days in space according to the SJ-10 satellite schedule but LCPT may last for many months. Therefore, proper selection of the samples of LDHs suspensions is essential to making behavior of LCPT observable in the 15day period. As a result, samples with different SC were tested for 15 days in the ground basis experiment and the relevant changes in samples were observed through crossed polarizers. The results and discussion will be presented in the following sections.

\section{Experimental Section}

The $\mathrm{Mg}_{2} \mathrm{Al}$ LDHs sample was synthesized by coprecipitation at $\mathrm{pH}$ 9.5-10. A mixed solution of magnesium and aluminum chlorides was prepared with a molar ratio of $\mathrm{Mg}^{2+} / \mathrm{Al}^{3+}=2: 1$, and the total concentration of the 
Fig. 2 Polarized light photographs of the $28 \mathrm{wt} \%$ $\mathrm{Mg}_{2} \mathrm{Al} \mathrm{LDHs}$ suspension: a just prepared, b 5 days, $\mathbf{c} 12$ days, d 15 days
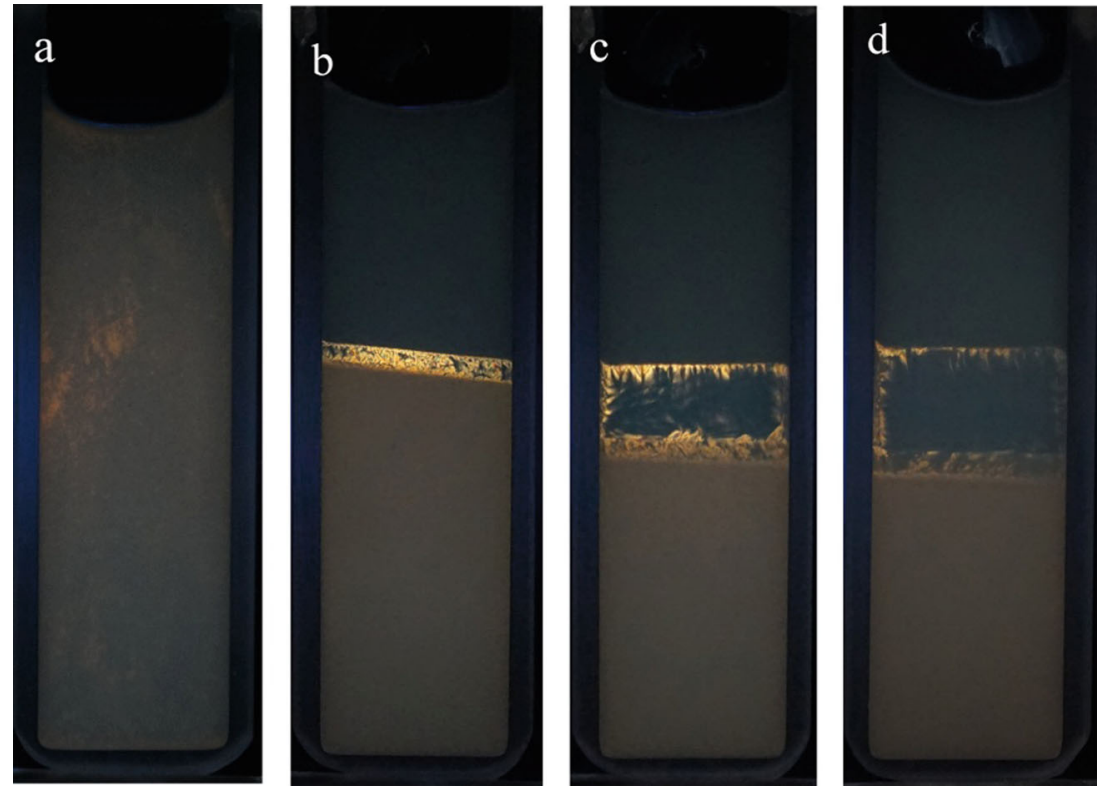

$\mathrm{Mg}$ and $\mathrm{Al}$ ion content was $0.5 \mathrm{M}$. Then, diluted ammonia solution $(20 \mathrm{vol} \%)$ was slowly added to the mixed solution under vigorous stirring. After coprecipitation for $45 \mathrm{~min}$, the suspension was washed with deionized water. The filter cake was then placed in a preheated oven, followed by hydrothermal treatment at a temperature of 80 ${ }^{\circ} \mathrm{C}$ for $24 \mathrm{~h}$. The as-obtained suspension was concentrated in vacuum until the solid content was up to $40 \mathrm{wt} \%$. The samples were tested further after dilution to the required concentration.

The chemical composition of the $\mathrm{Mg}_{2} \mathrm{Al}$ LDHs was $\mathrm{Mg}_{0.68} \mathrm{Al}_{0.36}(\mathrm{OH})_{2.30} \mathrm{Cl}_{0.18} \cdot 0.78 \mathrm{H}_{2} \mathrm{O}$ based on chemical analysis. The morphology and particle size distribution of the $\mathrm{Mg}_{2} \mathrm{Al}$ LDHs particles were characterized by JEM100CXII (JEOL, Japan) transmission electron microscopy (TEM). As shown in Fig. 1, most of the particles have hexagonal shape with different diameter $\mathrm{D}$ and thickness T. The corner-to-corner diameter of 500 particles was measured to obtain the average. The equation of the polydispersity in the diameter $\left(\sigma_{\mathrm{D}}\right)$ was defined as $\sigma_{\mathrm{D}}=\sqrt{\left.<D^{2}>-<D\right\rangle^{2}} /<D>$. Here, $<\mathrm{D}>$ was the average corner-to-corner diameter. The thickness (L) of the $\mathrm{Mg}_{2} \mathrm{Al}$ LDHs particles was measured by an atomic force microscope (AFM) which was recorded on a Digital Instrument Nanoscope IIIa Multimode system (Santa, Barbara, CA) in tapping mode. Similarly, the average thickness of the particles $(<\mathrm{L}>)$ was defined as the average of $\mathrm{L}$, and the polydispersity in the thickness $\left(\sigma_{\mathrm{L}}\right)$ was $\sigma_{\mathrm{L}}=\sqrt{\left.<L^{2}>-<L\right\rangle^{2}} /<L>$. The characteristics of $\mathrm{Mg}_{2} \mathrm{Al}$ LDHs particles were listed in Table 1.

\section{Results and Discussion}

\section{Phase Behavior of $\mathrm{Mg}_{2} \mathrm{Al}$ LDHs Suspensions in Gravity}

The birefringence of suspensions of the $\mathrm{Mg}_{2} \mathrm{Al}$ LDHs platelets was examined between crossed polarizers in sealed cuvettes with a width of $1 \mathrm{~mm}$. Onsager (1949) indicated that when the aspect ratio of particles was above 5, there would be a LCPT at high enough concentration. In fact, on a time scale of 15 days, the dispersions showed complex phase behavior with different suspension concentration (SC) and rest time. At low particle concentration ( $\mathrm{SC}<23 \mathrm{wt} \%$ ), the suspensions were in the isotropic state. On increasing the SC to above $23 \mathrm{wt} \%$, the dispersions showed an I-N transition. When $23 \mathrm{wt} \%<\mathrm{SC}<30 \mathrm{wt} \%$, the suspensions showed a shear-induced birefringence after preparation (Fig. 2a), indicating a highly-ordered directional alignment of particles. We defined the critical concentration of the formation of the shear-induced birefringence as $\mathrm{C}_{\mathrm{s}}$. Within five days, the shear-induced structure gradually relaxed, and then a nematic phase developed at the top of that structure. The nematic phase separated into two clearly different ordered phases with time. At last, coexistence of four phases was found, including an opaque isotropic top phase, a birefringent middle phase, a faint birefringence new phase and a sediment layer of larger platelets resulting from the high polydispersity. It should be pointed out that the dark of the major birefringent phase was due to the homeotropic alignment of $\mathrm{Mg}_{2} \mathrm{Al} \mathrm{LDHs}$ particles. On increasing the SC further to above $32 \mathrm{wt} \%$, a gel was formed 


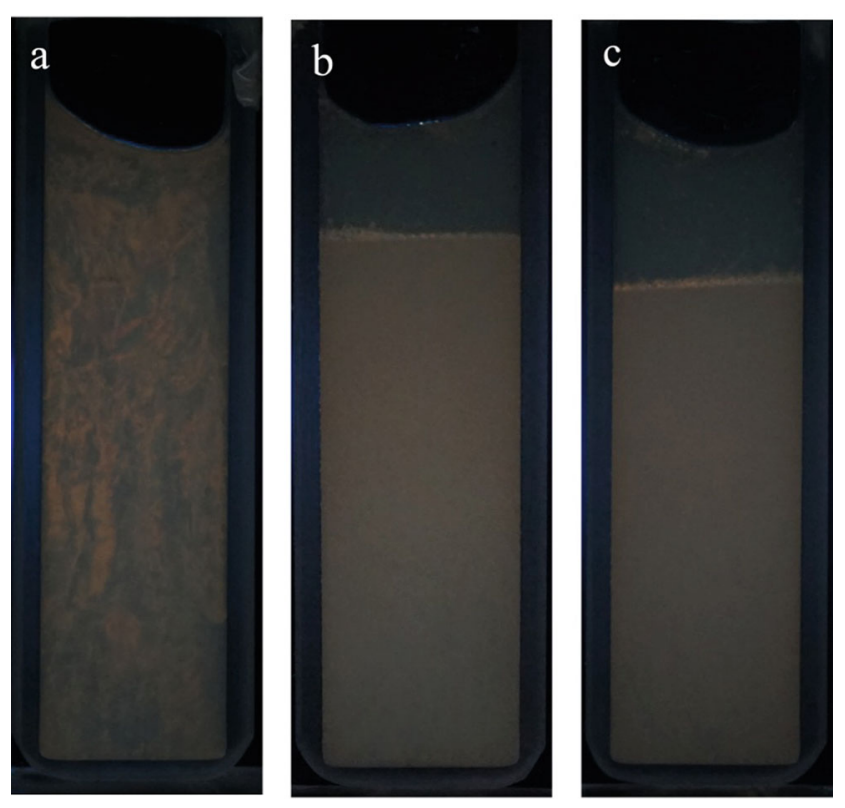

Fig. 3 Polarized light photographs of the $32 \mathrm{wt} \% \mathrm{Mg}_{2} \mathrm{Al} \mathrm{LDHs}$ suspension: a just prepared, b 12days, c 15days

which can be judged from the typical texture (Mourad et al. 2009) (Fig. 3a). We defined the critical concentration of the formation of a gel as $\mathrm{C}_{\mathrm{G}}$. The LCPT slowed down because of the formation of the gel network. After 12 days, we observed the phase transition proceeded with sedimentation of bright liquid crystal droplets (Fig. 3b). Three-phase coexistence appeared at last, including an isotropic top phase, a middle nematic phase and a sediment layer.

The particle size polydispersity increases significantly due to different aggregation degree of $\mathrm{Mg}_{2} \mathrm{~A} 1$ particles. The slow sedimentation rate of small particles or aggregates permits rearrangement into ordered phase, but larger ones settle down from the top to the bottom quickly and always form amorphous sediments instead (Davis et al. 1989). At last, there will be a density gradient and particle size fractionation in a single test tube. In suspensions of highly polydisperse goethite particles, the formation of smectic phase was attributed to a synergistic effect of Brownian motion, sedimentation, and fractionation by Vroege (2006). If colloidal gibbsite $\mathrm{Al}(\mathrm{OH})_{3}$ platelets were under a high gravitational force, an opal-like columnar crystal could be easily formed in only one day, a process which could take years under normal gravity (Van Beek et al. 2007). Obviously, the formation of ordered phase was greatly influenced by the strength of gravity, and Van der Beek attributed this fast formation of columnar opals to the forced sedimentation which was accompanied by fractionation. Consequently, the influence of gravity-induced sedimentation on LCPT can be considered in two aspects.
On one hand, the average size dramatically increases downwards the bottom due to the different sedimentation rate of particles or aggregates. Therefore, the polydispersity will decrease through the fractionation in size, which benefits the orientationally ordered arrangement of particles. On the other hand, it is possible to induce a high particle concentration on the bottom in gravity. For this reason, the critical concentration of liquid crystalline phase transition $\left(C_{L}\right)$ may be reached, and the phase transition occurs more easily near the bottom but above the layers of amorphous sediment.

\section{On the Anticipation of Phase Behavior of $\mathrm{Mg}_{2} \mathrm{Al} \mathrm{LDHs}$ Suspensions in Microgravity}

Under normal gravity condition, the concentration gradient and fractionation undoubtedly facilitates the formation of an ordered phase. For I-N phase separation in rods suspensions in the earth gravitational field, the longer rods tend to orient first and form a nematic phase because their larger excluded volume is strengthened by sedimentation (Leferink op Reinink et al. 2013), while the shorter rods still remain behind in the isotropic phase (Van Den Pol et al. 2008). However, to our knowledge, the density and particle size is uniformly distributed in microgravity, and the LCPT is not favored in this condition. Therefore, we make the following prediction.

Samples that do not show an ordered phase in gravity will also be incapable of undergoing a phase transition in microgravity. Taking the lack of concentration gradient and fractionation into consideration, we think the $\mathrm{C}_{S}$ may be still below the $\mathrm{C}_{\mathrm{L}}$. Therefore, the LCPT may appear in microgravity when $\mathrm{C}_{\mathrm{S}}<\mathrm{SC}<\mathrm{C}_{\mathrm{G}}$. As for samples in a gel state, the rearrangement of particles may be hindered by the formation of network. As a result, the LCPT will not occur in microgravity.

\section{Selected Samples for the Upcoming Experiment in the SJ-10 Satellite}

The consideration of the sample selection for the mission of LCPT on SJ-10 satellite is based on the pilot study of LCPT described above. Because of the limitation of experiment condition, only six samples are allowed to be placed in SJ-10 satellite. Therefore, we select six samples from those which show LCPT according to the ground test. We divide the selected samples into two groups. According to the previous section, liquid crystal phase may appear between $\mathrm{C}_{\mathrm{S}}$ and $\mathrm{C}_{\mathrm{G}}$. Therefore, we choose five samples from this concentration range. To verify our prediction, another sample comes from concentration above $\mathrm{C}_{\mathrm{G}}$. 


\section{Conclusion}

We conducted a pilot study of the LCPT in polydisperse aqueous suspensions of $\mathrm{Mg}_{2} \mathrm{~A} 1 \mathrm{LDHs}$ under normal gravity condition by birefringence observations for the space program SJ-10 satellite. We found that with the increase of particle concentration, the suspensions showed different phase behavior of isotropic, four-phase coexistence and three-phase coexistence in 15days. The phase transitions were attributed to the concentration gradient and fractionation caused by gravity. Finally, we described how to select samples suitable for the mission of SJ-10 project according to the ground-based experiment and our anticipation for the upcoming microgravity experiments

Acknowledgments We are grateful for the National Natural Science Foundation of China (Grant 21333005). We also thank the editor and reviewers for the useful comments and suggestions.

\section{References}

Bates, M.A., Frenkel, D.: Influence of polydispersity on the phase behavior of colloidal liquid crystals: A Monte Carlo simulation study. J. Chem. Phys 109(14), 6193-6199 (1998)

Byelov, D.V., Mourad, M.C.D., Snigireva, I., Snigirev, A., Petukhov, A.V., Lekkerkerker, H.N.W.: Experimental observation of fractionated crystallization in polydisperse platelike colloids. Langmuir 26(10), 6898-6901 (2010)

Chowdhury, P.R., Bhattacharyya, K.G.: Ni/Ti layered double hydroxide: Synthesis, characterization and application as a photocatalyst for visible light degradation of aqueous methylene blue. Dalton Trans. 44(15), 6809-6824 (2015)

Davis, K.E., Russel, W.B., Glantschnig, W.J.: Disorder-to-order transition in settling suspensions of colloidal silica: X-ray measurements. Science 245(4917), 507-510 (1989)

Fonseca, D.M., Méheust, Y., Fossum, J.O., Knudsen, K.D., Parmar, K.P.S.: Phase diagram of polydisperse Na-fluorohectoritewater suspensions: A synchrotron small-angle x-ray scattering study. Phys. Rev. E: Stat. Nonlinear Soft Matter Phys. 79(2), 021402 (2009)

Hansen, E.L., Jabbari-Farouji, S., Mauroy, H., Plivelic, T.S., Bonn, D., Fossum, J.O.: Orientational order in a glass of charged platelets with a concentration gradient. Soft Matter 9(42), 9999-10004 (2013)

Hu, W.R., Zhao, J.F., Long, M., Zhang, X.W., Liu, Q.S., Hou, M.Y., Kang, Q., Wang, Y.R., Xu, S.H., Kong, W.J., Zhang, H., Wang, S.F., Sun, Y.Q., Hang, H.Y., Huang, Y.P., Cai, W.M., Zhao, Y., Dai, J.W., Zheng, H.Q., Duan, E.K., Wang, J.F.: Space Program SJ-10 of Microgravity Research. Microgravity Sci. Technol. 26(3), 159169 (2014)

Kleshchanok, D., Holmqvist, P., Meijer, J.M., Lekkerkerker, H.N.W.: Lyotropic smectic b phase formed in suspensions of charged colloidal platelets. J. Am. Chem. Soc 134(13), 59855990 (2012a)

Kleshchanok, D., Meijer, J.M., Petukhov, A.V., Portale, G., Lekkerkerker, H.N.W.: Sedimentation and depletion attraction directing glass and liquid crystal formation in aqueous platelet/sphere mixtures. Soft Matter 8(1), 191-197 (2012b)
Kofke, D.A., Bolhuis, P.G.: Freezing of polydisperse hard spheres. Phys. Rev. E: Stat. Phys. Plasmas Fluids 59(1), 618-622 (1999)

Kuijk, A., Byelov, D.V., Petukhov, A.V., Van Blaaderen, A., Imhof, A.: Phase behavior of colloidal silica rods. Faraday Discuss. 159, 181-199 (2012)

Landman, J., Paineau, E., Davidson, P., Bihannic, I., Michot, L.J., Philippe, A.M., Petukhov, A.V., Lekkerkerker, H.N.W.: Effects of added silica nanoparticles on the nematic liquid crystal phase formation in beidellite suspensions. J. Phys. Chem. B 118(18), 49134919 (2014)

Leferink Op Reinink, A.B.G.M., Van Den Pol, E., Byelov, D.V., Petukhov, A.V., Vroege, G.J.: Ageing in a system of polydisperse goethite boardlike particles showing rich phase behaviour. J. Phys-Condens Mat. 24(46) (2012)

Leferink op Reinink, A.B.G.M., van den Pol, E., Petukhov, A.V., Vroege, G.J., Lekkerkerker, H.N.W.: Phase behaviour of lyotropic liquid crystals in external fields and confinement. Eur. Phys. J-Spec Top 222(11), 3053-3069 (2013)

Lettinga, M.P., Kang, K., Holmqvist, P., Imhof, A., Derks, D., Dhont, J.K.G.: Nematic-isotropic spinodal decomposition kinetics of rodlike viruses. Phys. Rev. E-Stat Nonlin Soft Matter Phys. 73(1), 011412 (2006)

Liu, S., Zhang, J., Wang, N., Liu, W., Zhang, C., Sun, D.: Liquidcrystalline phases of colloidal dispersions of layered double hydroxides. Chem. Mater 15(17), 3240-3241 (2003)

Mourad, M.C.D., Byelov, D.V., Petukhov, A.V., De Winter, D.A.M., Verkleij, A.J., Lekkerkerker, H.N.W.: Sol-gel transitions and liquid crystal phase transitions in concentrated Aqueous suspensions of colloidal gibbsite platelets. J. Phys. Chem. B 113(34), 1160411613 (2009)

Onsager, L.: The Effects of Shape on the Interaction of Colloidal Particles. Ann. N.Y. Acad. Sci 51(4), 627-659 (1949)

Palberg, T.: Crystallization kinetics of colloidal model suspensions: Recent achievements and new perspectives. J. Phys-Condens Mat. 26(33), 333101 (2014)

Van Beek, D.D., Radstake, P.B., Petukhov, A.V., Lekkerkerker, H.N.W.: Fast formation of opal-like columnar colloidal crystals. Langmuir 23(23), 11343-11346 (2007)

Van Den Pol, E., Thies-Weesie, D.M.E., Petukhov, A.V., Vroege, G.J., Kvashnina, K.: Influence of polydispersity on the phase behavior of colloidal goethite. J. Chem. Phys. 129(16), 164715 (2008)

Van Der Beek, D., Lekkerkerker, H.N.W.: Liquid crystal phases of charged colloidal platelets. Langmuir 20(20), 8582-8586 (2004)

Veerman, J.A.C., Frenkel, D.: Phase diagram of a system of hard spherocylinders by computer simulation. Phys. Rev. A 41(6), 3237 3244 (1990)

Veerman, J.A.C., Frenkel, D.: Phase behavior of disklike hard-core mesogens. Phys. Rev. A 45(8), 5632-5648 (1992)

Verhoeff, A.A., Lekkerkerker, H.N.W.: Direct observation of columnar liquid crystal droplets. Soft Matter 8(18), 4865-4868 (2012)

Verhoeff, A.A., Wensink, H.H., Vis, M., Jackson, G., Lekkerkerker, H.N.W.: Liquid crystal phase transitions in systems of colloidal platelets with bimodal shape distribution. J. Phys. Chem. B 113(41), 13476-13484 (2009)

Vis, M., Wensink, H.H., Lekkerkerker, H.N.W., Kleshchanok, D.: Nematic and lamellar liquid-crystalline phases in suspensions of charged silica-coated gibbsite platelets. Mol. Phys 113(9-10), 1053-1060 (2015)

Viveros-Méndez, P.X., Gil-Villegas, A., Aranda-Espinoza, S.: Monte Carlo computer simulation of sedimentation of charged hard spherocylinders. J. Chem. Phys. 141(4), 044905 (2014)

Vroege, G.J., Thies-Weesie, D.M.E., Petukhov, A.V., Lemaire, B.J., Davidson, P.: Smectic liquid-crystalline order in suspensions of highly polydisperse goethite nanorods. Adv. Mater 18(19), 25652568 (2006) 
Wang, N., Liu, S., Zhang, J., Wu, Z., Chen, J., Sun, D.: Lamellar phase in colloidal suspensions of positively charged LDHs platelets. Soft Matter 1(6), 428-430 (2005)

Wijnhoven, J.E.G.J., Van't Zand, D.D., Van Der Beek, D., Lekkerkerker, H.N.W.: Sedimentation and phase transitions of colloidal gibbsite platelets. Langmuir 21(23), 10422-10427 (2005)

Yang, A.J.M., Fleming Iii, P.D., Gibbs, J.H.: Theory for the influence of gravity on liquid-vapor interfaces. J. Chem. Phys. 67(1), 74-80 (1977)
Zhang, F., Du, N., Song, S., Liu, J., Hou, W.: Mechano-hydrothermal synthesis of Mg2Al-NO3 layered double hydroxides. J. Solid State Chem 206, 45-50 (2013)

Zhang, J., Luan, L., Zhu, W., Liu, S., Sun, D.: Phase behavior of aqueous suspensions of $\mathrm{Mg} 2 \mathrm{Al}$ layered double hydroxide: The competition among nematic ordering, sedimentation, and gelation. Langmuir 23(10), 5331-5337 (2007)

Zocher, H.: Über freiwillige Strukturbildung in Solen. (Eine neue Art anisotrop flüssiger Medien). Z. Anorg. Allg. Chem. 147(1), 91110 (1925) 